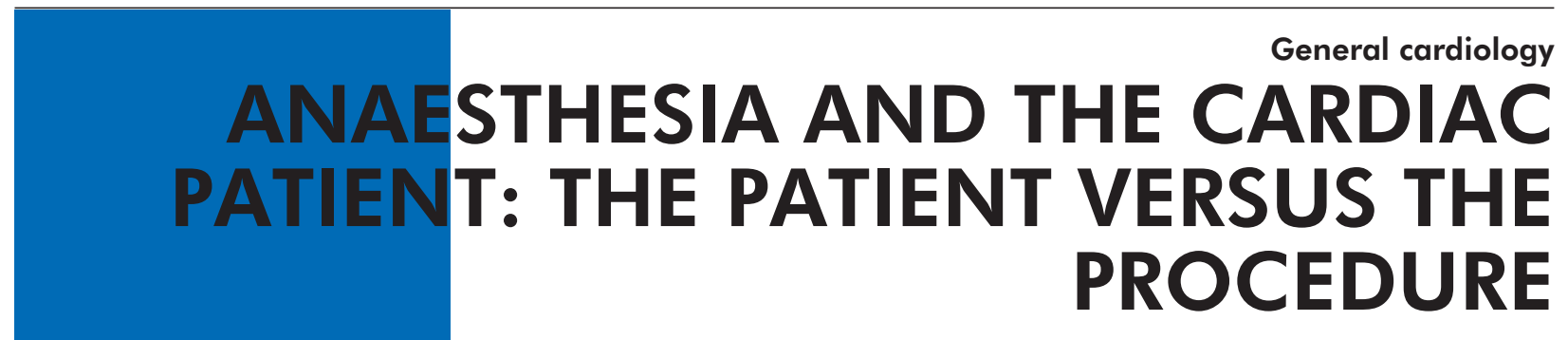

James B Froehlich, Kim A Eagle

Heart 2002;87:91-96

$\mathrm{F}$ or patients undergoing elective surgery, the most common cause of significant morbidity and mortality is occurrence of complications related to cardiac disease. ${ }^{1}$ It is estimated that approximately one million patients undergoing surgery each year in the USA suffer a perioperative myocardial infarction. ${ }^{1}$ This is particularly true for those with previous coronary disease and those facing higher risk surgery. Because of this fact, a great deal of research has focused on assessing cardiac risk before elective surgery. Less attention has been paid to methods of modifying the risk of cardiac complications attending surgery through medication use or other strategies. The risk of cardiac complications engenders a sense of conflict in that the patient perceives surgery as a threatening foe to be overcome: the patient versus the procedure. We would like to change that paradigm, and encourage an appreciation for the risk inherent to the patient, rather than

to the procedure itself. That is, preoperative evaluation of the patient's risk, versus the procedure's risk.

During the past 10-20 years, the assessment of cardiac risk before surgery evolved a great deal. Initially, the focus was on appropriate identification of surgical procedures that carried high risk. The focus then shifted to identifying those patient factors associated with increased risk of cardiac complications during surgery. Several technical advances were made during this time, including the introduction of imaging stress tests to assess cardiac ischaemia, such as dobutamine echocardiogram, dobutamine thallium, and adenosine or dipyridamole thallium testing. All of these modalities have been shown to identify patients at increased risk for cardiac complications of surgery. Cardiac catheterisation has also been used as a screening modality before elective surgery, though this has not been shown to be cost effective, especially given the low overall incidence of severe coronary artery disease. Studies performed during this time period also delineated the clinical factors that identify patients at increased risk of cardiac complications. More recently, efforts have been made to combine both clinical evaluation and testing in the most efficient and appropriate manner to identify patients at risk of cardiac complications. Finally, recent studies have addressed the effectiveness of medications or interventions to decrease risk in high risk patients.

Preoperative cardiac evaluation has several goals:

- evaluate and assess perioperative cardiac risk, and provide this information to both patient and surgeon for decision making purposes

- optimise appropriateness of preoperative testing and/or intervention

- to the extent possible, adjust care in order to decrease operative risk

- given the prevalence of coronary disease and its complications, assess and intervene to modify long term risks for cardiovascular disease.

We discuss below the current state of preoperative cardiac evaluation and interventions to decrease perioperative cardiac risk, and offer an approach to the preoperative assessment and perioperative care of patients with cardiac disease, focusing on risk reduction.

\title{
CLINICAL EVALUATION
}

Correspondence to James B Froehlich MD, UMass Memorial Medical Center, University of Massachusetts Medical School, Division of Cardiovascular Medicine, University Campus, 55 Lake Avenue North, Worcester, MA 01655, USA

froehlij@ummhc.org
Historically, the preoperative assessment of patients before elective surgery was based almost entirely on the clinical evaluation and examination. The American Society of Anesthesiology has used the ASA physical status classification system (1963) to grade perioperative risk. This classification could identify those at extremely high risk of complications from surgery, but did not offer much sensitivity in assessing patients' risk. The patients in level IV or V were at extremely elevated risk, but patients categorised in level III constituted a very wide spectrum of risk and comorbid disease. Furthermore, the ASA classification system does not focus on cardiac risk per se. It offers no consideration for the presence or absence of serious coronary disease in otherwise asymptomatic or undiagnosed patients.

Lee Goldman, then a resident at Massachusetts General Hospital, conducted a study that identified clinical factors conferring elevated risk of surgical complications. ${ }^{2}$ By performing a 
multivariate logistic regression analysis of a wide range of clinical parameters on 1000 consecutive patients undergoing elective surgery at the Massachusetts General Hospital, Goldman and his colleagues identified clinical markers of increased risk, and appropriately weighted them based on the epidemiological risk they conferred. The Goldman grading system allowed an estimate of the weighted risk of perioperative cardiac complications based on the presence or absence of clinical factors including the history of recent myocardial infarction, presence of congestive heart failure, critical aortic stenosis, significant non-cardiac organ failure or disease, urgency of surgery, and advanced age. The presence of these factors, particularly when added together, correlated with elevated risk. However, the majority of patients studied did not have markers of high risk and the index proved to be insensitive for discriminating risk in patients who would be considered intermediate in risk. The Goldman index did not include evaluation by objective stress testing, nor does it allow one to infer a plan for appropriate further steps in the evaluation process.

Several other studies have confirmed the utility of clinical evaluation in identifying patients at increased risk of significant coronary disease. L'Italien and others reviewed the clinical risk factors of patients undergoing elective vascular surgery at Massachusetts General Hospital, University of Massachusetts Medical Center, and the University of Vermont Medical Center, and analysed these clinical risk assessments with the results of thallium functional testing, also done before surgery. ${ }^{3}$ This group initially identified a small list of clinical factors that conferred risk based on multivariate logistic regression analysis. These clinical factors are advanced age, a history of diabetes, myocardial infarction, angina, or congestive heart failure. This group's findings, corroborated by other groups, revealed that the absence of any of these clinical markers of risk conferred a very low risk of complications of surgery (3\% in this study). Likewise, the presence of one or two of these factors conferred a moderately increased risk $(8 \%)$ and the presence of three or more a high risk of death or myocardial infarction during vascular surgery (18\% in this study).

Paul and colleagues reviewed an extensive database of cardiac catheterisation results on 878 consecutive patients undergoing elective vascular surgery at the Cleveland Clinic. ${ }^{4}$ They reviewed these same five clinical markers of risk, and observed that the presence of three or more of these clinical markers was coincident with a high likelihood of three vessel or left main coronary artery disease. Similarly, the absence of any of these markers of risk was coincident with a very low likelihood of having severe coronary artery disease on catheterisation. Taken together, these studies of clinical markers of cardiac risk suggest that patients who are properly evaluated, and have none of these clinical markers of risk, have a very low likelihood of suffering cardiac complications of surgery. This finding has recently been corroborated in clinical trials of the effect of perioperative $\beta$ blockade on cardiac complications.

\section{NON-INVASIVE TESTING}

The introduction of sensitive non-invasive tests for coronary artery disease, particularly pharmacologic stress tests that require no treadmill exercise, has greatly influenced the preoperative assessment of cardiac risk. Several early studies demonstrated a very high sensitivity of these tests for identifying patients at increased risk of perioperative cardiac complications. Most impressively, these results have been repeatedly duplicated by a large number of investigators. In an important work on the subject, Boucher and others demonstrated that thallium testing before elective vascular surgery accurately identified those patients who suffered cardiac complications of surgery. ${ }^{5}$ Furthermore, those patients with a normal thallium study had a very low incidence of cardiac complications. This was followed by several other studies, which demonstrated essentially similar results. Taken together, the clinical studies of thallium testing before vascular surgery have shown strikingly consistent results. These are a very high sensitivity (between $85-100 \%$ ), but a fairly low specificity for the identification of patients who suffer cardiac complications of surgery. For this reason, the negative predictive value of thallium is quite high, better than $95 \%$, even combining all current clinical studies. The positive predictive value, however, is quite low because of the low specificity (a problem of false positive tests in lower risk patients). This makes thallium stress testing a reassuring test when negative, but clinically confusing when positive. Such results highlight the fact that thallium testing is inappropriate as a uniform screening test, particularly when applied to "low risk" individuals.

Fewer studies have examined dobutamine echocardiogram as a preoperative screening modality; however, the results are quite similar to those found with thallium testing. There is similar sensitivity with the same problem of relatively low specificity. At institutions that have established proficiency at dobutamine echocardiogram testing, the results are considered interchangeable with thallium testing. Dobutamine echocardiography has the advantage of providing information regarding valvar structure and function.

Exercise tolerance testing, without cardiac imaging, also has an important role in screening for cardiac risk. Exercise tolerance, combined with electrocardiographic interpretation (assuming a normal baseline ECG), has great prognostic power for the patient with known or suspected coronary disease. Similarly, the ability to achieve maximum predicted heart rate without ECG confers a low risk for cardiac complications of elective surgery. Because it evaluates exercise tolerance and gives an idea of the level of stress that may induce inducible ischaemia, exercise testing is generally preferable to pharmacologic testing, particularly for long term prognostication.

Because of the relatively non-specific nature of functional testing, it is best employed as a component of an organised programme for cardiac risk evaluation. Proper clinical assessment of pre-test probability of significant coronary disease will allow more prudent use and interpretation of ischaemia testing.

Invasive testing has been proposed as a screening modality for patients undergoing high risk surgery-for example, peripheral vascular reconstructions. Hertzer and colleagues reported from the Cleveland Clinic on the use of routine catheterisation on 1000 consecutive patients scheduled for vascular surgery. ${ }^{6}$ Although they reported a high incidence of patients with severe coronary disease, requiring coronary bypass grafting, subsequent review of the data suggests that most of those patients with coronary disease sufficiently severe to warrant revascularisation could be identified on clinical grounds. This, and the expense and risk of routine catheterisation, have led most to consider clinical and functional assessment as initial screening for cardiac risk. 


\section{METHODS FOR LOWERING PERIOPERATIVE CARDIAC RISK \\ Coronary bypass surgery}

Recently, attention has turned to evaluating the effectiveness of methods for intervening to lower risk of cardiac complications during elective surgery. Coronary revascularisation is one such intervention. A retrospective review by Eagle and colleagues of the CASS (coronary artery surgery study) registry data supports such a protective effect. ${ }^{7}$ These data demonstrate that patients undergoing elective vascular surgery, who had previously undergone coronary artery bypass grafting, did better than control patients who had similar amounts of coronary disease, but no surgical coronary revascularisation. This type of analysis does not take into consideration the cumulative risk of both coronary and peripheral revascularisation, and so does not necessarily argue for prophylactic surgical coronary revascularisation before elective peripheral vascular surgery. But it does suggest a protective effect of prior coronary bypass surgery. Data from the Cleveland Clinic showed similar findings- that patients with a history of coronary artery bypass grafting, regardless of clinical risk factors, had lower perioperative cardiac complication rates surrounding vascular surgery than patients with coronary disease managed medically. These studies argue that a history of successful coronary artery bypass surgery confers a lower risk of cardiac complications surrounding elective surgery.

\section{Percutaneous coronary intervention}

The discovery of ischaemia on functional testing frequently leads to consideration of percutaneous revascularisation before elective vascular surgery. This practice has not been subjected to randomised controlled trials to assess its efficacy. Trials are currently underway for this purpose. Previous randomised studies comparing medical treatment with angioplasty in patients with stable coronary disease of limited severity have demonstrated an increased event rate in those patients undergoing angioplasty. The bulk of this increase came in the form of periprocedural complications. Retrospective studies reporting the rates of perioperative cardiac complications in patients who underwent previous preoperative angioplasty and/or coronary stent placement have shown very mixed results. Posner and colleagues reported a lower rate of cardiac complications among patients who underwent angioplasty before surgery compared with a group of patients with coronary artery disease managed medically. ${ }^{8}$ This study is uncontrolled for severity of disease or medical management, however. Massie and associates performed a case-control study comparing patients with abnormal thallium studies who did and did not undergo angiography before vascular surgery, and found no difference in event rates. Hassan and colleagues found similarly low rates of cardiac complications after non-cardiac surgery among patients in the BARI (bypass angioplasty revascularization investigation) study. ${ }^{10}$ This was equally true for patients who had undergone multivessel percutaneous coronary intervention, as for those who underwent coronary bypass surgery. Finally, Kaluza and colleagues reported a very high incidence of stent thrombosis, death, and myocardial infarction in patients undergoing non-cardiac surgery within two weeks of coronary stent placement. ${ }^{11}$ These data raise concern that the strategy of prophylactic, percutaneous coronary revascularisation before elective surgery may result in destabilisation of previously stable coronary disease which offsets the potential advantage of improving ischaemic thresholds of the heart by reducing severe, fixed coronary stenoses.

\section{Perioperative medical treatment}

Several recent studies have suggested that $\beta$ blockers decrease risk of perioperative complications. A randomised study by Mangano and colleagues evaluated brief courses of perioperative $\beta$ blockade in patients undergoing a variety of surgical procedures. ${ }^{12}$ The study was small, and demonstrated no difference in perioperative complication rate. However, over the succeeding two years, the patients who received this brief course of perioperative $\beta$ blockade had a lower incidence of cardiac events. This study did not control for medications between the two groups, but at least raises the question of a protective effect of perioperative $\beta$ blockade. A more recent study by Poldermans and associates randomised only clinically high risk patients undergoing elective, major vascular surgery, to the $\beta$ blocker bucindolol or placebo. ${ }^{13}$ This study demonstrated a significant reduction in perioperative cardiac events, both fatal and non-fatal, with the use of a $\beta$ blocker. These patients were given $\beta$ blocker treatment days or weeks before surgery. The $\beta$ blocker was titrated to a target dose of 10 mg per day, so long as the heart rate remained above 60 beats per minute. These studies, combined with previous investigations that show a protective effect of $\beta$ blockers for both ambulatory and perioperative ischaemia, support the hypothesis that perioperative $\beta$ blockade decreases cardiac risk among high risk patients.

Finally, several recent studies evaluated the effect of $\alpha$ receptor agonists in the perioperative period on the incidence of cardiac events. In a large randomised controlled trial of intravenous $\alpha_{2}$ agonist mivazerol during surgery, Oliver and colleagues compared outcomes during surgery in patients who had either a history of coronary artery disease, or the presence of significant risk factors. ${ }^{14}$ They found no significant effect in the patients undergoing non-cardiac surgery in general, but a significant reduction in both cardiac events and death in the subset of patients undergoing vascular surgery. Mangano and colleagues reported the results of a randomised trial of the same agent in 300 patients undergoing non-cardiac surgery, and found no significant effect on cardiac events. ${ }^{15}$ These and other studies at least raise the possibility that intraoperative $\alpha$ agonists may reduce perioperative cardiac events.

These reports certainly raise hope for therapeutic intervention to lower perioperative risk of cardiac events. The initial $\beta$ blocker study of Poldermans and colleagues demonstrated benefit in a high risk cohort of patients undergoing vascular surgery. ${ }^{13}$ More recent data from the same group suggests benefit from $\beta$ blockade across all risk groups. This requires prospective trial validation. Currently, it seems quite reasonable to use the American College of Cardiology/American Heart Association (ACC/AHA) guidelines to assess risk, ${ }^{16}$ and consider $\beta$ blockade in any patients at increased risk not already taking them. The role of $\alpha$ agonists is less clear. The above mentioned studies suggest some benefit from their use in patients undergoing vascular surgery, but little is known about these patients, and what the indications for use of this agent would be.

\section{PUTTING IT ALL TOGETHER}

The past two decades have answered many questions about perioperative cardiac complications, and who is at increased risk for them. As discussed above, we have a good understanding of what constitutes a high risk patient, and what tests are useful in further defining risk. The ACC/AHA preoperative evaluation guidelines describe a method of integrating these data into an efficient, evidence based approach to evaluating cardiac risk. ${ }^{16}$ This approach incorporates three 
Clinical evaluation steps

(1) Emergency surgery

(2) Prior coronary revascularisation

(3) Prior coronary evaluation

(4) Clinical markers of risk?

(6) Surgery specific risks?

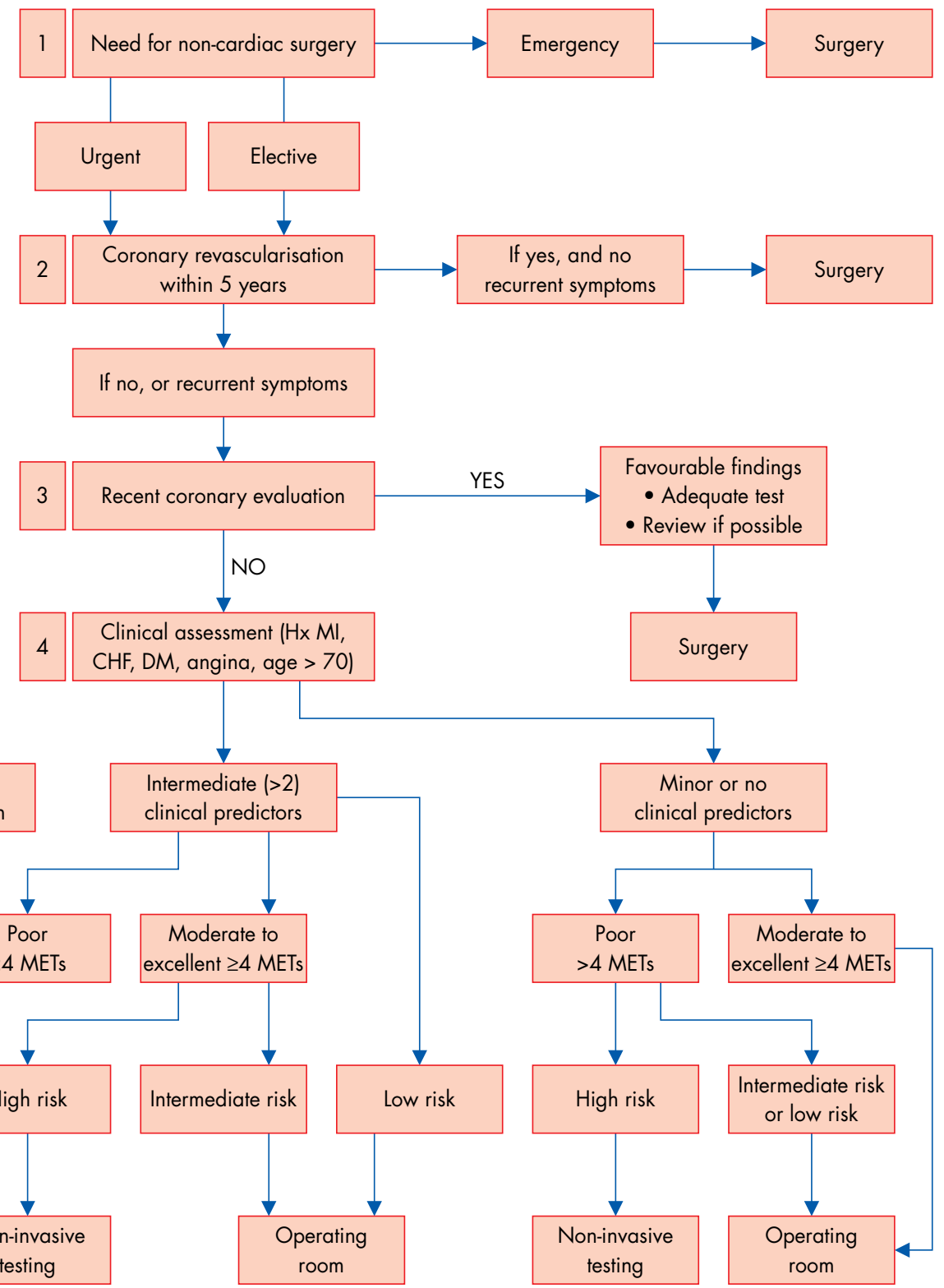

predictors $(A)$

Non-invasive testing or operating room

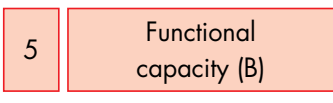

6 Surgical risk category (C)

Figure 1 Algorithm for cardiac risk assessment before non-cardiac surgery. $\mathrm{Hx} \mathrm{Ml}$, history of myocardial infarction; CHF, congestive heart failure; DM, diabetes mellitus.

steps: first, a clinical evaluation to determine the patient's likelihood of significant coronary disease, and perioperative cardiac event risk; second, selective use of non-invasive testing to further refine risk assessment; and third, intervention to further assess and/or modify cardiac risk. This approach should be taken with the patient's lifetime risk of cardiac disease manifestations as the end point, not just the perioperative period. The following algorithm outlines this approach (fig 1).

The first step in this algorithm is to determine urgency of the planned surgery. Obviously, emergent surgery should proceed without the delay of cardiac evaluation. Any surgical procedures not felt to be emergent allow for more thorough evaluation of cardiac risk. For patients who have undergone coronary revascularisation within the previous five years, without any recurrent symptoms of cardiac disease, further evaluation is probably unnecessary (step 2). If previous, recent, (within two years) adequate cardiac evaluation has taken place, without any change in clinical status, then there is usually no need to repeat
A. Major clinical predictors of increased cardiovascular risk

- Unstable coronary syndromes

- Recent myocardial infarction with evidence of important ischaemic risk by clinical symptoms or non-invasive study

- Unstable or severe angina (Canadian Cardiovascular Society class III or IV)

- Decompensated heart failure

- Significant arrhythmias

- High grade ventricular arrhythmias in the presence of underlying heart disease

- Supraventricular arrhythmias with uncontrolled ventricular rate

- Severe valvar disease

before surgery, if the results indicated low risk (step 3). Finally, a thorough clinical evaluation should be undertaken to determine if major markers of risk are present (for which 
B. Estimated functional capacity, based on daily activities

\section{MET}

- Can you take care of yourself?

- Eat, dress or use the toilet?

- Walk indoors around the house?

- Walk a block or two on level ground at 2-3 mph or $3.2-4.8 \mathrm{~km} / \mathrm{h}$ ?

- Do light work around the house like dusting or washing dishes?

4 METs

4 METs

- Climb a flight of stairs or walk up a hill?

- Walk on level ground at $4 \mathrm{mph}$ or $6.4 \mathrm{~km} / \mathrm{h}$ ?

- Run a short distance?

- Do heavy work around the house like scrubbing floors or lifting or moving heavy furniture?

- Participate in moderate recreational activities like golf, bowling, dancing, doubles tennis, or throwing a baseball or football?

- Participate in strenuous sports like swimming, singles tennis, football, basketball, or skiing?

$>10$ METs

METs, metabolic equivalents

\section{Risk stratification for non-cardiac surgical}

\section{procedures}

Major (reported cardiac risk often $>5 \%$ )

- Emergent major operations, particularly in the elderly

- Aortic and other major vascular

- Peripheral vascular

- Anticipated prolonged surgical procedures associated with large fluid shifts and/or blood loss

Intermediate (reported cardiac risk 1-5\%)

- Carotid endarterectomy

- Head and neck

- Intraperitoneal and intrathoracic

- Orthopaedic

- Prostate

Low* (reported cardiac risk generally $<1 \%$ )

- Endoscopic procedures

- Superficial procedure

- Cataract

- Breast

*Do not generally require further preoperative cardiac testing

cardiac catheterisation should be considered), or if any of the five clinical markers of risk are present (history of myocardial infarction, diabetes mellitus, congestive heart failure, angina, age $>70$ years). A decision about stress testing is based on the clinical markers of risk present, the patient's functional capacity by history, and the expected cardiovascular stress posed by noncardiac surgery (fig l).

In this way, a systematic approach, based on the current literature and validated prediction tools, can guide the assessment of risk, and the prudent use of further diagnostic testing of cardiac risk before non-cardiac surgery. As stated above, this systematic approach does not rely on testing, but incorporates clinical evaluation with objective testing to define cardiac risk of non-cardiac surgery optimally.

\section{Key points}

- Exploit opportunity of preoperative evaluation to assess and intervene upon reversible cardiovascular risk factors

- Evaluation based on cardiac risk, not pending surgery.

- Utilise history, physical, and ECG findings to stratify clinical risk

- Further evaluation (for example, stress testing, catheterisation), based on clinical evaluation, and probability of disease

- Use stress testing to modify, not co-opt, pre-testing likelihood of disease

- Decision regarding stress testing, cardiac catheterisation, or revascularisation, based on algorithm

- $\beta$ Blockade indicated for higher risk patients undergoing vascular surgery.

\section{REFERENCES}

1 Mangano DT, Goldman L. Preoperative assessment of patients with known or suspected coronary disease. N Engl J Med 1995;333:1750-6.

Excellent review of the topic.

2 Goldman L, Caldera DL, Nussbaum SR, et al. Multifactorial index of cardiac risk in non-cardiac surgical procedures. N Engl J Med 1977;297:845-50.

- Seminal work, identifying for the first time the magnitude and nature of the impact on perioperative cardiac risk of several important clinical findings.

3 L'Italien GJ, Paul SD, Hendel RC, et al. Development and validation of a Bayesian model for perioperative cardiac risk assessment in a cohort of 1,081 vascular surgical candidates. J Am Coll Cardiol 1996;27 779-86

- This study shows the relative roles of clinical and radiological evaluation of patients before vascular surgery, and the value of combining the two to improve accuracy and decrease testing.

4 Paul SD, Eagle KA, Kuntz KM, et al. Concordance of preoperative clinical risk with angiographic severity of coronary artery disease in patients undergoing vascular surgery Circulation 1996:94:1561-6.

- Confirmatory study, documenting the power of clinical risk factors to predict severity of coronary disease.

5 Boucher CA, Brewster DC, Darling RC, et al. Determination of cardiac risk by dipyridamole-thallium imaging before peripheral vascular surgery. N Engl J Med 1985;312:389-94.

6 Hertzer NR, Beven EG, Young JR, et al. Coronary artery disease in peripheral vascular patients: a classification of 1000 coronary angiograms and results of surgical management. Ann Surg 1984;199:223-32.

- Early, single centre report of findings on routine cardiac catheterisation in patients undergoing vascular surgery

7 Eagle KA, Rihal CS, Mickel MC, et al. Cardiac risk of noncardiac surgery: influence of coronary disease and type of surgery in 3368 operations. CASS Investigators and University of Michigan Heart Care Program. Coronary artery surgery study. Circulation 1997; 96:1882-7.

8 Posner KL, Van Norman GA, Chan V. Adverse cardiac outcomes after noncardiac surgery in patients with prior percutaneous transluminal coronary angioplasty. Anesth Analg 1999:89:553-60.

9 Massie MT, Rohrer M, Leppo JA, et al. Is coronary angiography necessary for vascular surgery patients who have positive results of dipyridamole thallium scans. J Vasc Surg 1997;25:975-82; discussion 982-3.

10 Hassan SA, Hlatky MA, Boothroyd D, et al. Outcomes of non-cardiac surgery after coronary bypass surgery or coronary angioplasty in the bypass angioplasty revascularization investigation (BARI). Am J Med (in press).

- BARI study data suggesting low rates of cardiac events after either coronary bypass surgery or multi-vessel coronary angioplasty before non-cardiac surgery.

11 Kaluza GL, Joseph J, Lee JR, et al. Catastrophic outcomes of non-cardiac surgery soon after coronary stenting. J Am Coll Cardiol 2000;35: 1288-94

- One of few observations about the important question of timing of surgery after percutaneous coronary intervention (PCI). This study suggests significant risk associated with stopping antiplatelet agents within 2-4 weeks of $\mathrm{PCl}$.

12 Mangano DT, Layug EL, Wallace A, et al. Effect of atenolol on mortality and cardiovascular morbidity after noncardiac surgery. Multicenter study of perioperative ischemia research group. N Engl J Med 1996;335: 1713-20. 
13 Poldermans D, Boersma E, Bax JJ, et al. The effect of bisoprolol on perioperative mortality and myocardial infarction in high-risk patients undergoing vascular surgery. Dutch echocardiographic cardiac risk evaluation applying stress echocardiography study group. N Engl J Med 1999:341:1789-94.

14 Oliver MF, Goldman L, Julian DG, et al. Effect of mivazerol on perioperative cardiac complications during non-cardiac surgery in patients with coronary heart disease. The European mivazerol trial (EMIT).
15 Anon. Perioperative sympatholysis. Beneficial effects of the alpha 2-adrenoceptor agonist mivazerol on hemodynamic stability and myocardial ischemia, MeSPI -Europe research group. Anesthesiology 1997;86:346-63.

16 Eagle KA, Brundage BH, Chaitman BR, et al. Guidelines for perioperative cardiovascular evaluation for non-cardiac surgery. Circulation. 1996;93:1278-317.

- Current ACC/AHA guidelines for cardiac evaluation prior to non-cardiac surgery. Contains a very thorough review of the entire English language literature. 